\title{
PRACTICAL TRACKING SYSTEM OF INTELLIGENT WORKSHOP PRODUCT BASED ON WIRELESS SENSOR NETWORK
}

\author{
Baozhou Zheng ${ }^{1 *}$, Yaqiong Zhu ${ }^{2}$ \\ ${ }^{1}$ College of Sciences, Henan Agricultural University, Zhengzhou 450002, China \\ ${ }^{2}$ Henan Institute of Finance \& Banking, Zhengzhou 451416, Henan, China \\ *Corresponding author: Baozhou Zheng \\ Email: zhengbzscience2013@126.com
}

\begin{abstract}
To make the wireless sensor network used for intelligent workshop products tracking system, firstly, the moving target tracking technology of wireless sensor networks was introduced and the target positioning technique based on ranging was emphatically analysed, which laid the technical foundation for the construction of moving target tracking system of wireless sensor network. Then, the extended Kalman filter algorithm with less calculation and high tracking accuracy and implementation steps of untraced Kalman algorithm were analysed and summarized. $\mathrm{C}++$ programming language was used to transform untraced Kalman filtering algorithm into intelligent workshop product practical tracking algorithm module. Moreover, the two algorithms were used in the workshop product tracking system based on wireless sensor network. The result showed that the system could realize the tracking effect of high precision and low power consumption. To sum up, the comparison of many groups of experiments also proves the performance difference of these two algorithms in the practical application.
\end{abstract}

Keywords: Wireless Sensor Networks, Intelligent Workshop Products, Moving Target Tracking, untraced Kalman Filter.

\section{Introduction}

Target tracking technology has an important application value in both military and civil fields [1]. In 1937, the first tracking radar station (SCR-28) appeared in the world [2]. Since then, with the continuous development and progress of sensor technology, target tracking systems based on radar, laser, infrared and satellite appear more and more in various fields. Moving target tracking is a hot topic in many scientific research institutes at home and abroad, which involves advanced technologies such as control, signal processing and communication.

Among them, this technology is also widely used in the intelligent workshop product tracking system.

Wireless sensor network (WSN) is a set of sensor nodes with sensor detecting, communication and event processing capabilities deployed in the monitoring area. These nodes form a multi-hop and self-organizing network system by wireless communication way [3-5]. It is put forward and studied by the US military for military needs. With the rapid development of wireless communication technology, micro computer technology, system on chip and low-power embedded device, the application field of wireless sensor network is wider and wider.
Because of its wide coverage and self-organizing ability, wireless sensor network has made great changes in information sensing field by adopting the advantages of wireless communication, low energy consumption and high reliability [6].

With the rise and progress of wireless sensor network research, how to locate and track moving targets using wireless sensor networks has become an important research topic of target tracking [7]. The nodes consisting of wireless sensor network has the characteristics of small volume, low price and low energy consumption. Nodes can transmit information through wireless communication and ad hoc network. These features make the moving target tracking system based on wireless sensor network have obvious advantages than traditional moving target tracking system [8].

Although the moving target tracking based on wireless sensor network has many advantages, the nature of the wireless sensor network is different from the ordinary network. The wireless sensor network itself has a large number of nodes, nodes are prone to failure, energy is constrained and transmission is unreliable.

The detection ability, computing ability, storage ability and so on of nodes consisting of wireless sensor network are seriously limited. All of these put forward challenge for the design and implementation of moving target tracking system based on wireless sensor network. 


\section{Methodology \\ 2.1 Basic content of moving target tracking in Wireless Sensor Networks}

Moving target tracking based on wireless sensor network mainly consists of three phases: detection, positioning and notification and each phase corresponds to the corresponding technology.

Among them, the detection stage is to choose a variety of detection methods to determine whether the moving target appears in the detection area. The commonly used detection techniques include radio signal intensity detection, ultrasonic detection, acoustic detection, vibration detection, infrared detection and laser detection. The positioning stage integrates multiple sensor information obtained in detection stage to determine the state of moving target. The commonly used method of this phase has three-side localization, dual detection and triangle detection.

According to these methods, the coordinate position of the target can be determined. In addition, the target data is recorded and used to filter and estimate the state and moving trajectory of target.
The circular stage is the process of each sensor node information exchange, which broadcasts the moving target current state information and estimated trajectory, notifies and wakes up sensor nodes near the estimated track to join the moving target tracking process.

The following simply illustrates the basic idea of single target tracking. Set the single moving target entering the well deployed wireless sensor networks detection region. The sensor nodes in the detection area can detect the moving targets, and then the sensor nodes detecting moving target forms the detection information into data packets and sends to the sink node. The sink node process all the packets received to form a comprehensive data packet sent to the host computer. The host computer receives the data, processes and calls the moving target tracking algorithm to estimate the coordinate position of target.

Along with the movement of the target, the sink node timely notifies the sensor nodes in the relevant detection area to join in the process of target tracking.

Figure 1 is the flow chart of the moving target tracking process.

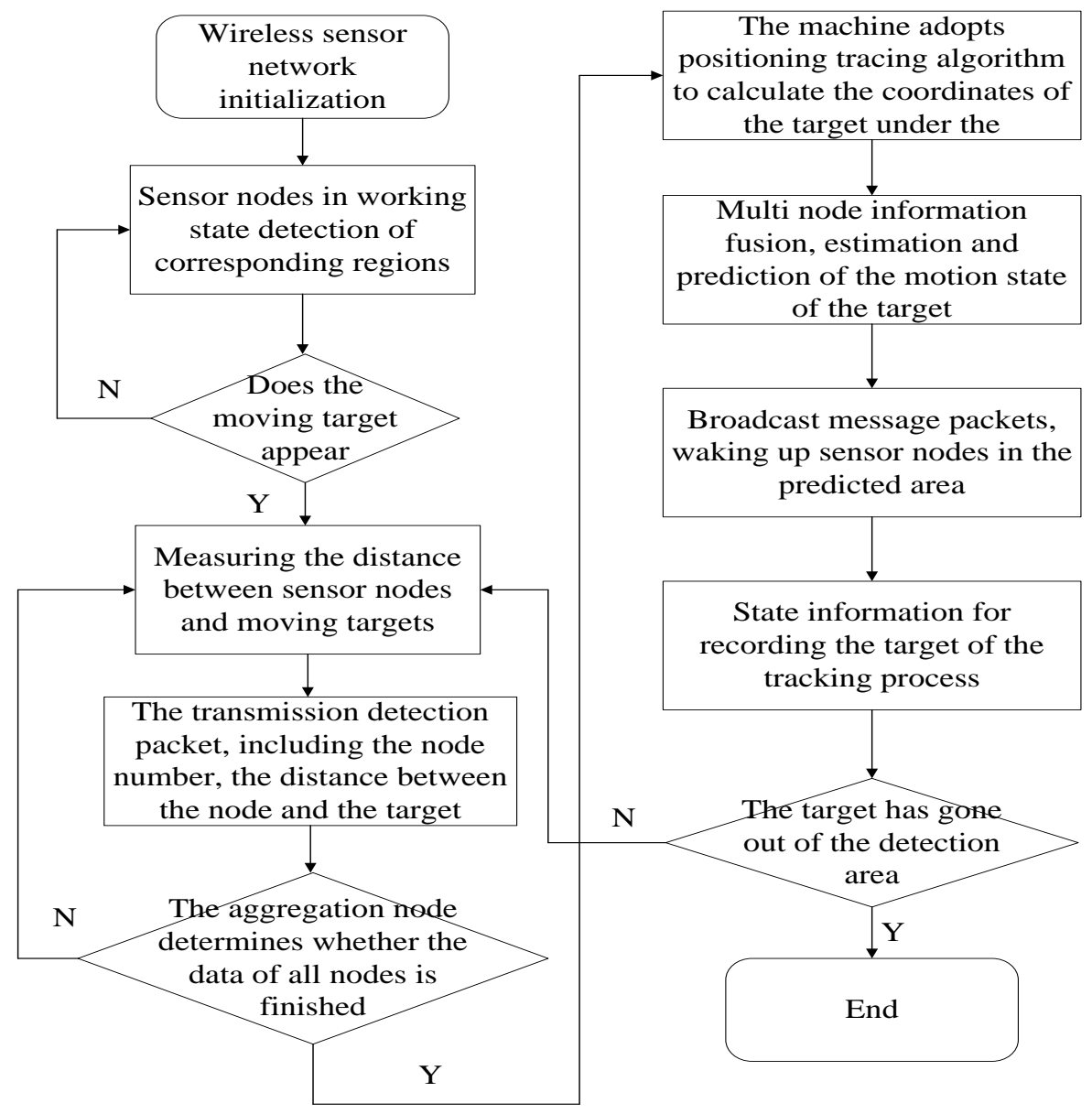

Figure. 1 Flow chart of target tracking process 


\subsection{Basic technology of moving target tracking in Wireless Sensor Networks}

The wireless sensor network consisting of sensor nodes is an equality network with no strict control centre, and the status of all nodes is equal. Based on the moving target tracking in wireless sensor networks, due to the sensor node energy, computing capacity and other constraints, a single sensor node cannot effectively track the target. It requires a number of sensor nodes for collaborative detection, and processes all available data. As a result, selforganization and routing problem of sensor nodes of wireless sensor network exists in the tracking process of moving target.

Localization in wireless sensor networks refers to the location information of nodes and targets obtained by self-organizing network, which is divided into node self-localization and target location.

The node self-localization is the process of determining the coordinate position of nodes in wireless sensor networks by means of manual calibration or various node self-localization algorithms.

The target location is, based on node selfpositioning of the nodes, with known nodes in the coordinate as the reference node, to determine the coordinate position of a target or an event in the detection range of wireless sensor networks. This paper focuses on target location and illustrates target location algorithm.

According to received signal strength indication (RSSI), the distance between signal transmitter node and receiver node is measured, which is called signal intensity ranging.

The transmitting power of the transmitting node is known, and the receiving power is calculated at the receiving node. The difference between the two is transmission power loss. Then, the transmission loss is converted to the distance by using the theoretical or practical signal propagation model. This method can use the following gradient model to calculate the distance:

$$
\begin{aligned}
& {[P(d)]_{d b m}=\left[P\left(d_{0}\right)\right]_{d b m}-10 \lg \left(\frac{d}{d_{0}}\right)} \\
& \frac{\gamma}{C^{2}} \frac{\partial^{2} p}{\partial t^{2}}-\frac{1}{a^{2}} \nabla^{2} p=\dot{S}
\end{aligned}
$$

In terms of non-ranging algorithm, there are mainly DV-Hop algorithm, SPA algorithm, centroid algorithm and convex programming algorithm. Among them, the three-side measurement method is shown in Figure 2.

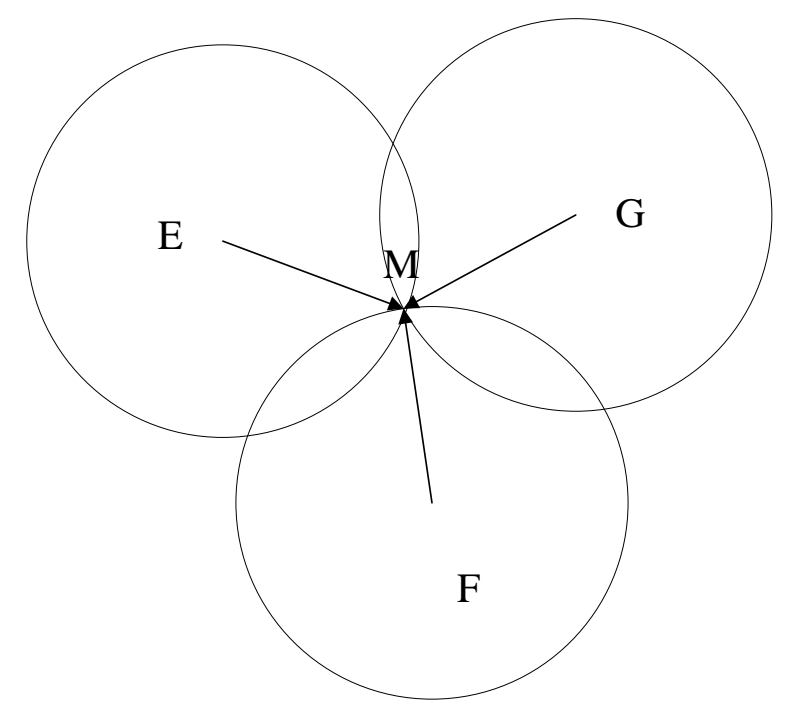

Figure. 2 Schematic diagram of three-side measurement

As shown in Figure 2, the coordinates of three nodes E, F and G are known as (xe, ye), (xf, yf) and (xg, yg). And the distance from them to the unknown node $\mathrm{M}$ is $\mathrm{de}$, $\mathrm{df}$ and $\mathrm{dg}$, respectively. Set the coordinate of unknown node $M$ to $(x, y)$, then from (2), ( $x, y)$ can be calculated and obtained.

$$
\left\{\begin{array}{l}
\sqrt{\left(x-x_{e}\right)^{2}+\left(y-y_{e}\right)^{2}}=d_{e} \\
\sqrt{\left(x-x_{f}\right)^{2}+\left(y-y_{f}\right)^{2}}=d_{f} \\
\sqrt{\left(x-x_{g}\right)^{2}+\left(y-y_{g}\right)^{2}}=d_{g}
\end{array}\right.
$$




\subsection{Untraced Kalman filter algorithm}

Set a nonlinear transformation $y=f(X)$. X suggests the random variable of $n$ dimension and the mean value and variance PX are known. The basic principle of untraced transformation is shown below:

Calculate $2 n+1$ Sigma points. Generate Sigma points set from the statistics amount of and PX of random vector $X$.

Although the extended Kalman filter algorithm solves the problem that the basic Kalman filtering algorithm can only be applied to linear systems, the extended Kalman filter algorithm must solve Jacobi matrix of the nonlinear function. For the system with complex model, the calculation is complex and prone to have errors. The extended Kalman filter algorithm also introduces the linearization error, which easily causes poor filtering effect for highly nonlinear system. Based on the above factors, in order to improve the filtering accuracy and efficiency of the filtering algorithm, a new filtering method must be found. The Untraced Kalman filter came into being. The untraced Kalman filtering is a new idea, which is derived from "the approximate operation of probability density distribution of nonlinear functions is simpler than that of nonlinear functions". Therefore, the application of sampling method to approximate nonlinear distribution for solving nonlinear filtering problem is an important feature of untraced Kalman filter. The untraced Kalman filtering algorithm is based on the untraced transformation (UT) and adopts the Kalman filtering algorithm framework, which is an improved Kalman filtering algorithm.

$$
\mathrm{X}_{i}=\left\{\begin{array}{c}
\overline{\mathrm{X}}+\left(\sqrt{\left.(n+\kappa) P_{\mathrm{X}}\right)_{i}}, i=1, \ldots n\right. \\
\overline{\mathrm{X}}-\left(\sqrt{\left.(n+\kappa) P_{\mathrm{X}}\right)_{i}}, i=n+1, \ldots 2 n\right. \\
\overline{\mathrm{X}}, i=0
\end{array}\right.
$$

$\kappa$ indicates the scale parameter and adjusting $\kappa$ can improve the filtering performance. The group of sample points $\mathrm{Xi}$ is used to approximately represent the Gauss distribution of the state $\mathrm{X}$.

Nonlinear transformation is conducted on Sigma point. $f\left({ }^{*}\right)$ nonlinear transformation is conducted on the generated points set $\{\mathrm{Xi}\}$ to obtain the transformed Sigma points set:

$$
\mathrm{Y}_{i}=f\left(X_{i}\right), i=0,1, \ldots 2 n
$$

Calculate the mean value and variance of $y$. First of all, the weighted computation of the points set $\{\mathrm{Yi}\}$ is carried out, and the mean value and variance of the output $y$ are obtained, which can be got from (5).

$$
\left\{\begin{array}{c}
\omega_{0}^{(m)}=\frac{\kappa}{n+\kappa} \\
\omega_{0}^{(c)}=\frac{\kappa}{n+\kappa}+\left(1-\alpha^{2}+\beta\right) \\
\omega_{i}^{(m)}=\omega_{i}^{(c)}=\frac{\kappa}{2(n+\kappa)}, i=1, \ldots 2 n
\end{array}\right.
$$

In the above formula, $\kappa=\alpha^{2}(n+\lambda)-n$, In the weighted computation of mean value and variance, we need to determine the value of parameters $\alpha, \beta$ and $\lambda$. Their value range is: $\alpha$ value range is a smaller positive number in $1 \mathrm{e}-4<\alpha<1 ; \beta$ is state distribution parameter. In Gauss distribution, $\beta=2$ is optimal. If the state variable is the single variable, $\beta=0$ is the best choice; $\lambda$ is generally set to 0 or $3-n$; in general, adjusting $\beta$ can improve the precision of estimated variance and reasonably adjusting $\alpha$ and $\lambda$ can improve the precision effect of estimated mean value. The schematic diagram of untraced transformation is shown in Figure 3.

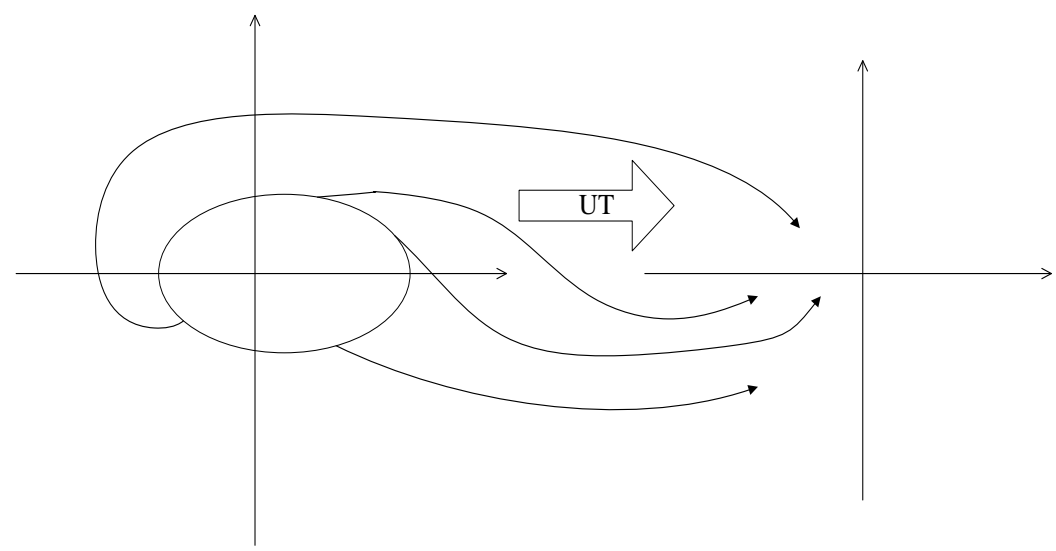

Figure. 3 Untraced transformation diagram 


\subsection{Experimental model of moving target tracking system}

In order to apply the extended Kalman filtering algorithm and the unscented Kalman filtering algorithm to the workshop product tracking system built in this paper, we set up an experimental model of moving target tracking system.

It is difficult to establish an accurate motion model of moving target in the practical application environment. Therefore, many scholars have put forward the moving target motion model under various reasonable assumptions. In the motion model of the moving target above, the uniform speed model is relatively simple, strong and convenient for computer processing.

In Cartesian coordinate system, the system state is set to $X(k)=\left[x(k), x^{\prime}(k), y(k), y^{\prime}(k)\right]^{T}$.

The state of system includes the location $x(k)$ and velocity $x^{\prime}(k)$ in $x$ direction. The random disturbance in moving target motion is expressed as $\mathrm{W}(\mathrm{k})$, and the uniform motion model of moving target is established as follows:

$$
\left\{\begin{array}{c}
x(k+1)=x(k)+v_{x}(k) T+\frac{1}{2} \omega_{x}(k) T^{2} \\
v_{x}(k+1)=v_{x}(k)+\omega_{x}(k) T \\
y(k+1)=y(k)+v_{y}(k) T+\frac{1}{2} \omega_{y}(k) T^{2} \\
v_{y}(k+1)=v_{y}(k)+\omega_{y}(k) T
\end{array}\right.
$$

For the sake of convenient expression, it is customary to write the state of the system as:

$$
X(k+1)=\phi X(k)+\Gamma W(k)
$$

$$
\begin{aligned}
& \begin{array}{llllll}
1 & \mathrm{~T} & 0 & 0 & \mathrm{~T}^{2} / 2 & 0
\end{array} \\
& \phi=\begin{array}{llll}
0 & 1 & 0 & 0 \\
0 & 0 & 1 & \mathrm{~T}
\end{array} \Gamma=\begin{array}{cc}
\mathrm{T} & 0 \\
0 & \mathrm{~T}^{2} / 2
\end{array} \\
& \begin{array}{llllll}
0 & 0 & 0 & 1
\end{array}, \quad 0 \quad \mathrm{~T} \\
& W(k)=\left[\omega_{x}(k), \omega_{y}(k)\right]^{T}, T=0.5
\end{aligned}
$$

So far, the experimental model of moving target tracking system has been established. From the model, we can know that the state equation of the system is linear, and the observation equation is nonlinear.

\section{Results and Discussion}

\subsection{Overall structure design of the intelligent workshop product tracking system in} wireless sensor network

The moving target tracking system based on wireless sensor network is composed of multiple sensor nodes, 1 converging node and a PC. The tracking target is workshop product, which realizes the detection and tracking of the moving target in the network coverage area. The sensor node location in the system is known and sensor nodes access the distance information of node and moving target by ultrasonic sensor. The sink node, through wireless sensor network, collects the distance information and corresponding node number (coordinate information) of each sensor node. Then, the collected information is uploaded to the PC; at the same time, processing software running on PC processes the acquired information and calls the target tracking algorithm to obtain the coordinate position of moving target. Moreover, the trajectory of moving target is real-time rendered to the display interface for monitoring the working state of each sensor node. The overall structure of the system is designed as shown in Figure 4.

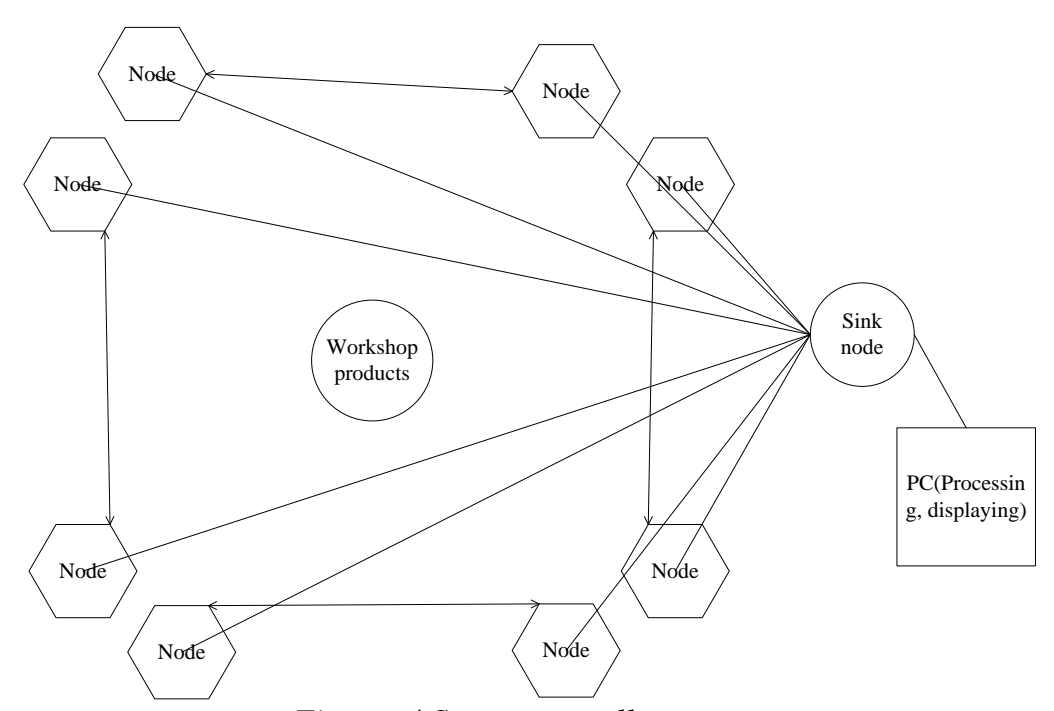

Figure. 4 System overall structure 


\subsection{System hardware platform}

The development of ZigBee wireless network needs related hardware and software. In hardware aspect, TI Company has launched a CC2530 chip which fully supports ZigBee2007 (ZigBee protocol) protocol, and has also developed the corresponding development kit. There are also many companies in the country that launch a ZigBee development kit based on CC2530 chips. The development kit based on CC2530 chip basically supports the ZigBee2007 protocol stack (also known as Z-stack) launched by TI Company. The development of sensor nodes in the system is based on the CC2530 development board launched by Net-bee technology. The hardware design of the development board is open, which is conducive to the construction of various applications of wireless sensor network nodes.

The intelligent workshop product is the object of system tracking. This paper makes a moving target based on E-puck moving robot. The E-puck moving robot is mainly composed of DSPIC controller, interactive servo motor, all kinds of photoelectric sensors and power supply modules.
The E-puck moving robot can set the path and speed of its motion by programming, so it is very convenient to use it as a tracking object.

In this paper, a wireless sensor network moving target tracking system, composed of multiple sensor nodes, is designed to track the product of the workshop. The system hardware is mainly composed of 8 common sensor nodes and a sink node. The sensor nodes are arranged in the measuring area set covering the four corners of the rectangular region of $1.2 \mathrm{~m} * 1.2 \mathrm{~m}$, used for measuring the distance between cylindrical moving target edge and the sensor node in the monitoring area. The sink node is connected with PC through the serial port (RS232 protocol). The measurement information of sensor nodes and sink sensor node can be dispatched. The information will be uploaded to the host computer at PC side. Subsequently, the host computer, through calling the function modules, iteratively estimates the coordinate position of workshop products and records motion trajectory of workshop products.

The final result of the system hardware platform is shown in Figure 5.

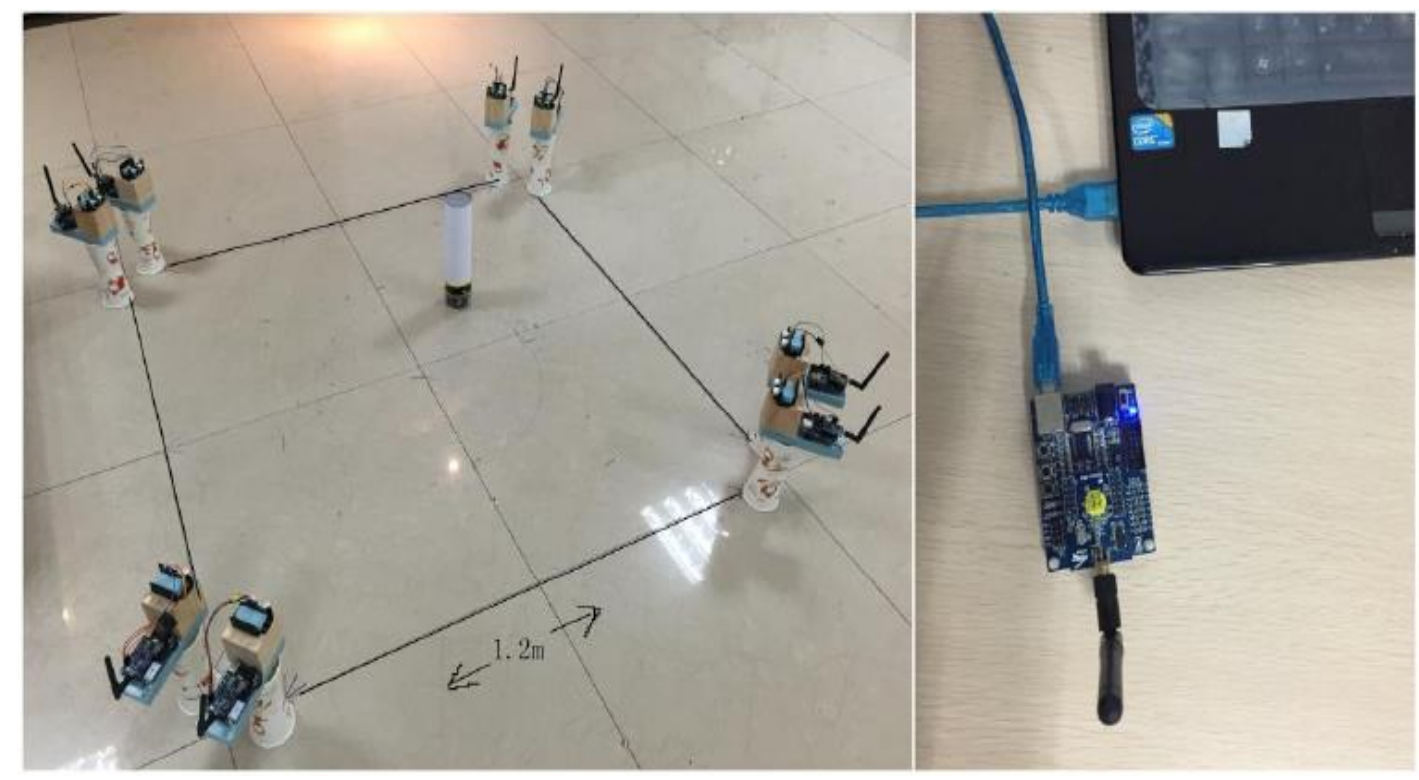

Figure. 5 System hardware platform

\subsection{System software framework}

Communication protocol is defined by a series of communication standards. Communication objects need to send or receive data according to the common communication standards, so as to ensure the accuracy of data transmission.

With the rapid development of wireless sensor networks, various wireless sensor network protocols are gradually standardized. Among them, a widely applied and widely promoted protocol is ZigBee. ZigBee, as a communication protocol, defines a series of communication specifications for wireless communications with short distance and low rate data transmission.

As shown in Figure 6, the ZigBee protocol specification can be divided into two parts. IEEE802.15.4 defines the physical layer and MAC level specification.

ZigBee alliance has developed network layer, security layer and application layer specification. The wireless network work band constructed by ZigBee is $868 \mathrm{MHz}, 915 \mathrm{Mhz}$ and $2.4 \mathrm{GHz}$, and the maximum transmission rate is $250 \mathrm{kbps}$ 


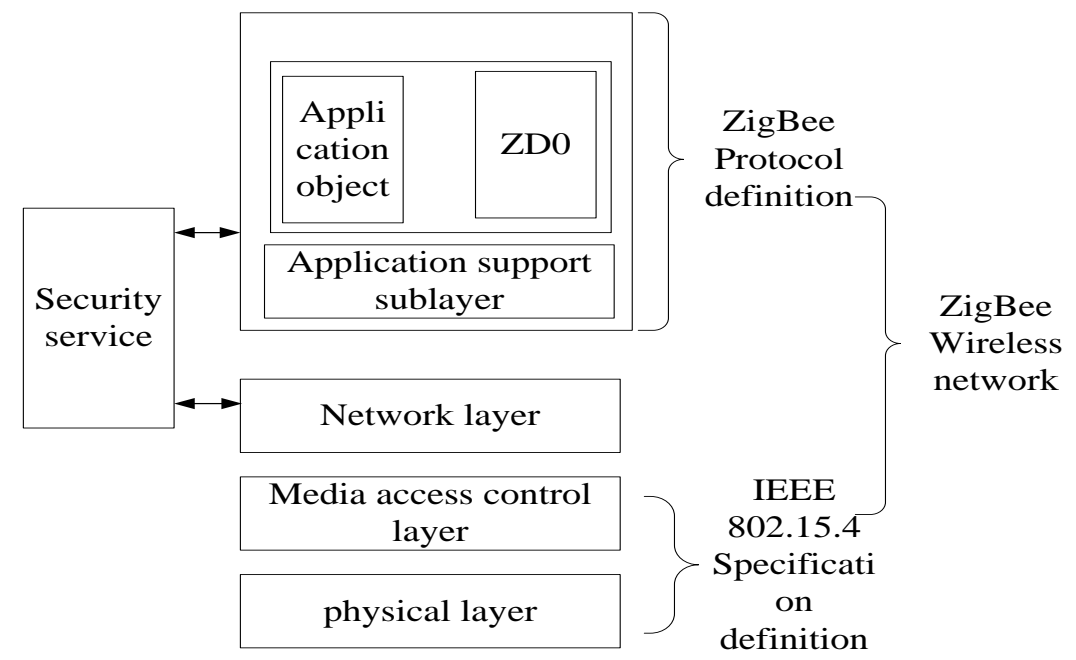

Figure. 6 Schematic diagram of ZigBee protocol layers

In the ZigBee protocol stack, OSAL is responsible for scheduling the operation of various task events, and when the event occurs, it calls the corresponding event processing functions. From this point of view, OSAL is a polling operating system based on event driven.
Event driven means a corresponding event handling after an event occurs and polling refers to a constant view of the occurrence of events.

The schematic diagram of the principle of OSAL works is shown in Figure 7.

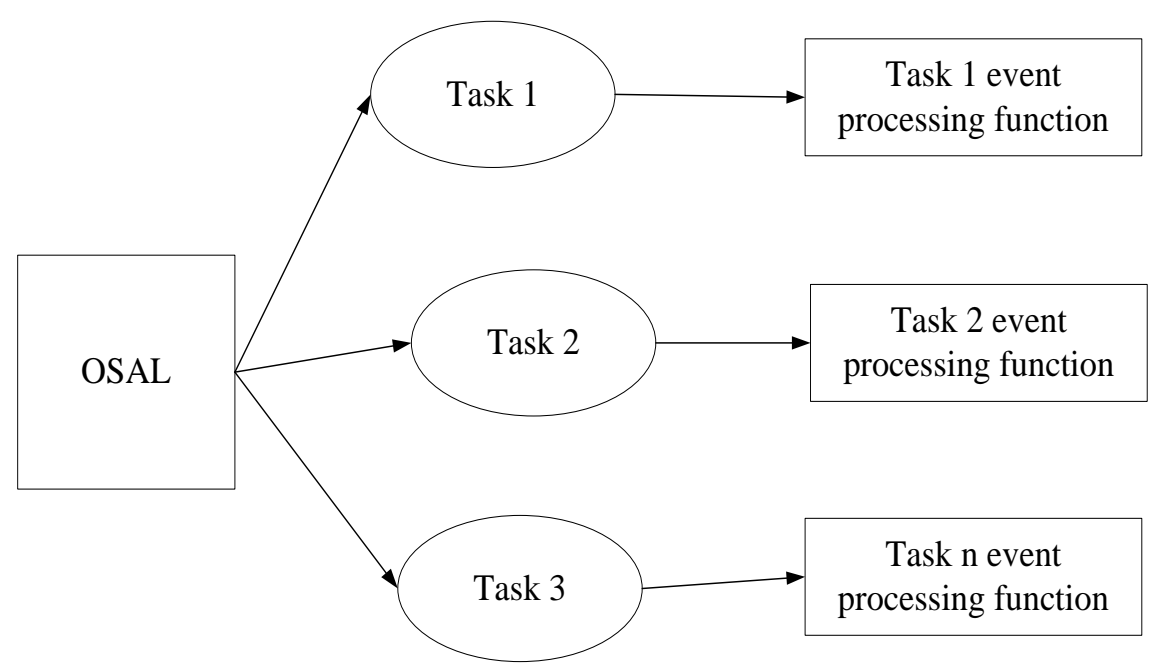

Figure. 7 Schematic diagram of OSAL working principle

\subsection{Measurement accuracy experiment of ultrasonic sensor}

Before the target positioning and tracking experiment, measure accuracy of ultrasonic sensor is the basic experiment. Through the measurement data of the ultrasonic sensor, the measurement error covariance $\mathrm{R}$ is calculated, which provides data support for the actual measurement error covariance $\mathrm{R}$ in EKF algorithm and UKF algorithm. The experimental environment is shown in Figure 8.

In this experiment, the tape is taken as the reference value of the distance, and the cylindrical plastic cylinder is used as the target.
The target is placed in the direction of $60 \mathrm{~cm}$, $120 \mathrm{~cm}$ and $180 \mathrm{~cm}$ in the direction of the ultrasonic sensor, which is measured 100 times at each place.

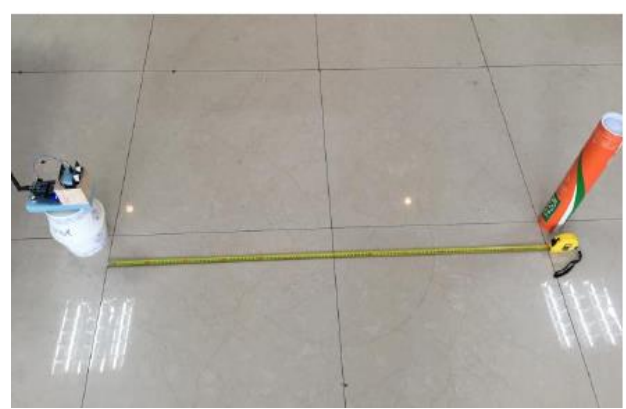

Figure. 8 Sensor precision measurement 
The experimental results are shown in Table 1 . It can be seen from the table, from $60 \mathrm{~cm}$ to $180 \mathrm{~cm}$, with the increase of measuring distance, average measurement error of ultrasonic sensor also began to change.

The average measurement error of $60 \mathrm{~cm}$ is $0.81 \mathrm{~cm}$, the average measurement error of $120 \mathrm{~cm}$ is $1.03 \mathrm{~cm}$ and the average measurement error of $180 \mathrm{~cm}$ is $2.45 \mathrm{~cm}$.
Through the analysis and calculation, the average error value of the ultrasonic sensor in the measuring range is about $1.50 \mathrm{cma}$ and the error covariance is 0.52 .

From the experimental results, it can be concluded that the KS103 ultrasonic sensor selected by the system has high ranging precision and meets the design requirements of the system.

Table 1 Experimental data of sensor measurement accuracy

\begin{tabular}{|c|c|c|c|}
\hline Number & Reference value $60 \mathrm{~cm}$ & Reference value $120 \mathrm{~cm}$ & Reference value $180 \mathrm{~cm}$ \\
\hline 1 & $59.1 \mathrm{~cm}$ & $120.8 \mathrm{~cm}$ & $182.5 \mathrm{~cm}$ \\
\hline 2 & $59.1 \mathrm{~cm}$ & $120.8 \mathrm{~cm}$ & $182.6 \mathrm{~cm}$ \\
\hline 3 & $59.1 \mathrm{~cm}$ & $120.2 \mathrm{~cm}$ & $182.5 \mathrm{~cm}$ \\
\hline 4 & $59.3 \mathrm{~cm}$ & $120.1 \mathrm{~cm}$ & $182.4 \mathrm{~cm}$ \\
\hline 5 & $59.1 \mathrm{~cm}$ & $120.3 \mathrm{~cm}$ & $182.5 \mathrm{~cm}$ \\
\hline 6 & $59.4 \mathrm{~cm}$ & $120.8 \mathrm{~cm}$ & $182.6 \mathrm{~cm}$ \\
\hline 7 & $59.1 \mathrm{~cm}$ & $120.5 \mathrm{~cm}$ & $182.5 \mathrm{~cm}$ \\
\hline 8 & $59.0 \mathrm{~cm}$ & $120.8 \mathrm{~cm}$ & $182.4 \mathrm{~cm}$ \\
\hline 9 & $59.2 \mathrm{~cm}$ & $120.9 \mathrm{~cm}$ & $182.6 \mathrm{~cm}$ \\
\hline 10 & $59.1 \mathrm{~cm}$ & $120.8 \mathrm{~cm}$ & $182.6 \mathrm{~cm}$ \\
\hline 11 & $59.3 \mathrm{~cm}$ & $120.0 \mathrm{~cm}$ & $182.3 \mathrm{~cm}$ \\
\hline$\ldots$ & $\ldots$ & $\ldots$ & $\ldots$ \\
\hline 100 & $59.2 \mathrm{~cm}$ & $120.4 \mathrm{~cm}$ & $182.4 \mathrm{~cm}$ \\
\hline
\end{tabular}

The three-point location is to locate the target through the distance information of the three ultrasonic sensors. The main purpose of this experiment is to evaluate the static positioning accuracy of the target tracking system. The ultrasonic sensors are placed in four corners of the monitoring area. The location and tracking algorithm is calculated by selecting the measurement information of three ultrasonic sensors nearest to the target, so as to the estimated coordinate position of the target.

The target is placed manually in each position of the monitoring area. In the experiment, the actual coordinate position of the target in the monitoring area is obtained by measuring the tape directly, the target coordinate position estimated by real-time moving target tracking algorithm in the upper computer is compared and the corresponding experimental results are recorded.

In this experiment, the deviation of coordinate values is used as the main evaluation index of the positioning accuracy of the evaluation system.
It needs to be explained that, in order to conveniently see the location and display results of the host computer, the display part has enlarged and translated the coordinate value $(\mathrm{x}, \mathrm{y})$ of the actual location result, and the coordinate value actually displayed is $\left(3^{*} \mathrm{x}-50,3^{*} \mathrm{y}-50\right)$ with unit of $\mathrm{cm}$.

The three-point localization experiment was carried out in 10 groups, and the record data were shown in Table 2.

It can be concluded from the table, the estimated value of $\mathrm{X}$ axis coordinate system and the $\mathrm{Y}$ axis and the target actual value of $X$ axis coordinates and the $\mathrm{Y}$ axis deviation was less than $2 \mathrm{~cm}$.

After calculation, the actual maximum deviation distance between the target coordinate estimated by the system and the actual target coordinate is less than $2.3 \mathrm{~cm}$, which is only $2.05 \%$ deviation relative to the monitoring area of $120 \mathrm{~cm}$.

It can be concluded that the target tracking system constructed based on wireless sensor network has good positioning accuracy. 
Table 2 Three-point positioning experimental data

\begin{tabular}{|c|c|c|c|c|c|c|}
\hline \multirow{2}{*}{ Number } & \multicolumn{2}{|c|}{ Real value $(\mathrm{cm})$} & \multicolumn{2}{c|}{ Estimated value(cm) } & \multicolumn{2}{c|}{ Coordinate deviation(cm) } \\
\cline { 2 - 7 } & X coordinates & Y coordinates & X coordinates & Y coordinates & X coordinates & Y coordinates \\
\hline 1 & 45 & 59 & 43.98 & 59.93 & 1.19 & 0.59 \\
\hline 2 & 59 & 59 & 59.88 & 60.12 & 0.63 & 0.91 \\
\hline 3 & 59 & 85 & 60.03 & 81.97 & 0.71 & 1.29 \\
\hline 4 & 36 & 60 & 36.21 & 60.21 & 1.01 & 0.66 \\
\hline 5 & 86 & 59 & 86.91 & 59.10 & 0.31 & 0.79 \\
\hline 6 & 77 & 77 & 74.59 & 76.98 & 1.38 & 0.28 \\
\hline 7 & 80 & 41 & 82.43 & 41.67 & 1.31 & 1.38 \\
\hline 8 & 40 & 40 & 42.06 & 37.92 & 0.58 & 0.96 \\
\hline 9 & 45 & 78 & 43.54 & 76.29 & 0.63 & 1.68 \\
\hline 10 & 69 & 45 & 72.04 & 47.04 & 1.58 & 0.81 \\
\hline
\end{tabular}

\section{Conclusion}

As a hot research technology, moving target tracking technology has been widely used in various fields. With the help of low cost, low power consumption, self-organization and high error tolerance of wireless sensor networks, moving target tracking based on wireless sensor networks also has broad application prospects. Based on existing wireless sensor network moving target tracking algorithm and moving target tracking technology, we designed a set of intelligent workshop product tracking system with low cost, low power consumption and high tracking accuracy.

The workshop products tracking system experimental platform based on wireless sensor network is designed and implemented. The wireless sensor network node is constructed by using ultrasonic sensor with low power consumption and high precision, and the system hardware platform construction, sensor nodes program design, sink node program design and PC software design are completed. At the same time, using the $\mathrm{C}++$ programming language, extended Kalman filter algorithm and unscented Kalman filtering algorithm are realized, respectively. And these two filtering algorithms are applied to the workshop product tracking experiment platform constructed. In the experiment platform, the performance of two kinds of filtering algorithms in the actual application environment is compared and evaluated. Moreover, the tracking effect of workshop product tracking system based on wireless sensor network is verified. However, in the face of the continuous enrichment and development of sensor technology, it will be a promising research direction how to apply the new sensor technology to moving target tracking and how to design a target tracking algorithm with lower computation and higher accuracy.

\section{References}

[1] Xie, S., \& Wang, Y. (2014). Construction of tree network with limited delivery latency in homogeneous wireless sensor networks. Wireless personal communications, 78(1), 231246.

[2] Borges, L. M., Velez, F. J., \& Lebres, A. S. (2014). Survey on the characterization and classification of wireless sensor network applications. IEEE Communications Surveys \& Tutorials, 16(4), 1860-1890.

[3] Tuna, G., Gungor, V. C., \& Gulez, K. (2014). An autonomous wireless sensor network deployment system using mobile robots for human existence detection in case of disasters. Ad Hoc Networks, 13, 54-68.

[4] Leu, J. S., Chiang, T. H., Yu, M. C., \& Su, K. W. (2015). Energy efficient clustering scheme for prolonging the lifetime of wireless sensor network with isolated nodes. IEEE communications letters, 19(2), 259-262.

[5] Al-Karaki, J. N., \& Kamal, A. E. (2004). Routing techniques in wireless sensor networks: a survey. IEEE wireless communications, 11(6), 6-28.

[6] Ramesh, M. V. (2014). Design, development, and deployment of a wireless sensor network for detection of landslides. Ad Hoc Networks, 13, 218.

[7] Misra, S., Chatterjee, S., \& Obaidat, M. S. (2017). On theoretical modeling of sensor cloud: A paradigm shift from wireless sensor network. IEEE Systems journal, 11(2), 1084-1093.

[8] Keskin, M. E., Altınel, İ. K., Aras, N., \& Ersoy, C. (2014). Wireless sensor network lifetime maximization by optimal sensor deployment, activity scheduling, data routing and sink mobility. Ad Hoc Networks, 17, 18-36. 